\title{
Transhumanismo Lado B: ¿Realidad que sobrepasa la ficción?
}

\author{
doi: $10.52749 /$ fh.v2i3.6
}

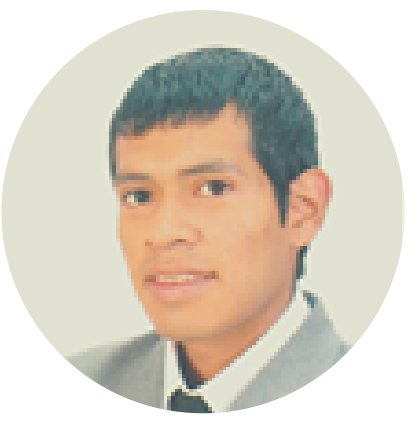

\section{WILDO SIANCAS}

Psicólogo por la Universidad San Ignacio de Loyola. Cuenta con estudios de maestría en Educación con mención en Psicología educativa. Se ha desempeñado como docente en organizaciones no gubernamentales orientadas al sector educativo y en psicología clínica infantil. Consultor independiente en metodología de la investigación. Miembro de la Sociedad Secular Humanista del Perú.

wildo_esmith_14@hotmail.com

Se habla y se considera que tanto la ciencia como el arte mantienen diferentes esferas de la realidad de manera independiente. Sin embargo, es necesario mencionar que tanto la literatura como la cinematografía (ambas manifestaciones artísticas) han denotado con creces el desarrollo, incluso visionario, de los avances científico-tecnológicos del contexto histórico-cultural del ser humano. Claros ejemplos de ello suelen ser las obras literarias de Julio Verne, tales como De la tierra a la luna (1865) y 20000 leguas de viaje submarino (1870), la serie de entregas de la Fundación escritas por Isacc Asimov (1942 -1922) e incluso los Códex politemáticos de Leonardo Da Vinci durante el renacimiento. Pero, a medida que se daba cabida a los beneficios y futuros avances científico-tecnológicos, junto a ellos, se concebían al unísono las distopías y sus diversas vertientes, las cuales llegaron al grado de plasmarse en el paradigma "High tech, Low life" propio del género literario Cyberpunk. El cine ha tratado de retratar aquel fenómeno de manera audiovisual, colocando como punto de partida las connotaciones estéticas, simbólicas y conceptuales de esta vertiente. Esta es la razón por la cual el objetivo del presente texto es continuar el trabajo expuesto en Transhumanismo Lado A y observar la otra cara de la moneda del Transhumanismo: El Lado B, esa contraparte del cassette que muy poco se toma en cuenta, pero que a través de ésta podemos obtener un panorama amplio del impacto de la vertiente transhumanista en las sociedades futuras. Si bien el análisis se realizará bajo la lupa del género Cyberpunk, a través de sus manifestaciones literarias y cinematográficas dentro de la cultura popular, al menos será un primer paso para conce- bir próximos espacios de diálogo acerca de este futuro tan distópico, pero no tan lejano a la realidad que conocemos.

\section{Complementos orgánicos exponenciales}

Una de las ideas que suele mantener el contexto literario de las obras Cyberpunk amerita el desarrollo amplio y vasto de las biotecnologías, así como el alto costo que implica acceder a sus mejoras y las consecuencias de no realizarlo. Podemos ver un ejemplo de ello en la película Los recolectores (2010) en la cual los órganos sintéticos trasplantados suelen hallarse bajo hipoteca, en donde si no cancelas la cuota monetaria a tiempo, este suele ser extraído del hospedero. Asimismo, en la película Gattaca (1997) se suele apreciar el uso de la ingeniería genética desde un enfoque determinista que aumenta las brechas de acceso a una óptima calidad de vida. Si bien estas dos obras cinematográficas presentan un panorama relevante en cuanto al desarrollo y manipulación de las biotecnologías dentro del dominio público de sus habitantes, es menester mencionar que el hilo de sus argumentos guarda relación con ciertas obras literarias como The repossession mambo de Eric García (2009) y Un mundo feliz de Aldous Huxley (1932), obras icónicas dentro de la literatura distópica y la ciencia ficción. En ambas tramas se trae a colación un análisis exhaustivo de lo que hoy en día conocemos como la Bioética aplicada al desarrollo de biotecnologías exponenciales, así como los futuros retos a los que se enfrentará la investigación, desarrollo e innovación $(I+D+i)$ dentro de este campo, tales como: la desigualdad en los niveles de cobertura de acceso a la salud, el auge de 
movimientos de biohackers radicales o incluso el nacimiento de niños editados genéticamente, como el caso de las dos niñas editadas genéticamente en China (Cárdenas, 2019; GBD, 2019; Wolinsky, 2016).

\section{Singularidad tecnológica incorpórea a la identidad humana}

Otro de los postulados que caracteriza la vertiente del Cyberpunk es la presentación de entidades artificiales e inorgánicas capaces de emular procesos cognoscitivos análogos al ser humano. Un ejemplo de ello se puede observar en los replicantes propios de la película Blade Runner (1982) los que, al ser entidades sintéticas orientadas a realizar trabajos forzados y riesgosos, manifiestan cierto grado de humanidad al intentar mantener su propia existencia a pesar del inminente final que les depara: el exterminio o la degradación con el devenir de los años. Este hilo argumentativo se mantiene en otras películas como Inmortal (2015), Transcendence: Identidad virtual (2014) o Chappie (2015), en donde manifestar cierto grado de consciencia, sea transferida, incorporada o resultado de alguna singularidad tecnológica, permite analizar hasta qué punto estos organismos sintéticos o híbridos lograrían concebir niveles de identidad incorpórea análogos al ser humano.

Aquel aspecto trae a colación las obras icónicas de este género, tales como ¿Sueñan los androides con ovejas eléctricas? De Philip K. Dick (1968) y Neuromante de William Gibson (1984), cuyas tramas abordan la decadencia de la vida y de la sociedad, así como diversos planteamientos éticos en cuanto a los androides y los límites entre lo real y lo artificial. Dimensiones que, actualmente, la Ciberpsicología se encarga de abordar a través del estudio de las interacciones sociales y los procesos de cambio en la conducta de los organismos inducidos por las nuevas tecnologías (Parsons, 2017). Estos aportes han permitido el desarrollo de arquitecturas emocionales en Inteligencia Artificial o incluso el desarrollo de bots capaces de brindar calidad informativa y conexión emocional al momento de interactuar con la audiencia (González \& González, 2017; González \& García, 2006). Pero estos avances también ponen en discusión el desarrollo de sesgos raciales y de género en algoritmos informáticos, procesos de discriminación logarítmica en selección de personal, accidentes en infraestructuras digitales, armas programables de destrucción masiva o aumento del desempleo en humanos (Mendoza, 2020; Páez \& Ramírez, 2020; Turchin \& Denkenberger, 2018).

\section{Sistemas ideológicos totalitarios}

En cuanto a los sistemas gubernamentales presentados a lo largo de la trama del género Cyberpunk $\mathrm{u}$ otras entregas relacionadas con la ciencia ficción, el auge de regímenes totalitarios que restringen el análisis crítico de los fundamentalismos del gobierno imperante son una manifestación clara del contexto social donde se hallan inmersos los personajes. Un ejemplo de ello suele ser la Fundación de Isacc Asimov (1951), cuya trilogía, aparte de realizar un aporte considerable como las tres leyes de la robótica, también manifiesta la reticencia de los gobernantes en cambiar su mismo proceder en el imperio. Ello bajo el paradigma del "non plus ultra" en cuanto al conocimiento científico humano. Aquel ideal acabaría sometiendo al gobierno de turno en su propia corrupción e inacción a pesar de contar con indicadores preestablecidos como la evidencia psicohistórica.

Lo anterior es una condición muy cercana a obras icónicas como El cuento de la criada de Margaret Atwood (1985), Fahrenheit 451 de Ray Bradbury (1953), Rebelión en la granja de George Orwell (1945) o 1984 del mismo autor (1949), cuyas adaptaciones a la pantalla grande han sido precedidas por directores como Volker Schlöndorff (1990), François Truffaut (1966), Michael Radford (1984), John Stephenson (1999), entre otros. En estas distopías, los sistemas de gobierno imperantes suelen tomar un control totalitario en la calidad de vida y desarrollo social de sus habitantes. A tal grado de hacer uso de diversos recursos como ejecuciones de opositores, seguimientos que violan la privacidad o incluso técnicas de manipulación para que los adeptos al régimen y sus disidentes oscilen entre el exterminio, la sumisión o el fanatismo ciego. Todo ello bajo la excusa de proteger el simbolismo trastocado de una falsa sensación de gobernabilidad consensuada que suele ser basada en Pseudoideologías fundamentalistas. Un indicador no muy lejano en la realidad, si observamos los acontecimientos actuales, tales como el golpe de estado en Myanmar, las protestas de Hong Kong en China, la toma del estado de Afganistán por parte de los talibanes, la situación actual de Corea del Norte y de Venezuela, las protestas en la isla de Cuba e incluso la coyuntura reciente en las últimas elecciones peruanas y los acontecimientos de no- 
viembre del 2020. Estos indicadores sociales lejos de reflejar el estandarte del ciberactivismo imperante, solo nos acercan a esta realidad presente dentro de la literatura y la cinematografía en su conjunto, de la cual el ser humano ve, pero no observa, oye, pero no escucha, actúa, pero no concientiza.

\section{Contextos líquidos de alta demanda}

Por último, dentro de los diversos contextos en donde se desarrollan este tipo de obras y filmaciones, la baja consideración del impacto humano en el medio ambiente y la explotación de los recursos a expensas del cambio climático, suelen ser parte de ese paisaje ficticio y que sentimos muchas veces lejano.Un ejemplo de ello sería La chica mecánica del escritor Paolo Bacigalupi (2009), en donde el calentamiento global realizó escarnios en la distribución de los combustibles fósiles, redujo el acceso a los cultivos de alimentos y las catástrofes o plagas suelen ser un escenario frecuente. Condición que en cierta medida es real, pues el Panel Intergubernamental del Cambio Climático manifestó, en el informe de este año, el crecimiento constante de $1.5^{\circ} \mathrm{C}$ en la temperatura de la tierra. Un factor que seguirá aumentando al menos hasta mediados de este siglo en todos los escenarios de emisiones considerados. Una realidad donde el aumento de la frecuencia e intensidad de las temperaturas extremas, las olas de calor marinas y las precipitaciones intensas serán parte de las futuras generaciones de este siglo (IPCC, 2021).

Asimismo, el mercantilismo corporativo que tiende a sobredimensionar el valor de las utilidades y sus datos a expensas del capital humano y de los usuarios. Un aspecto observado en la película El precio del mañana (2011), en donde el costo por seguir viviendo el día suele ser demasiado alto en comparación a lo que se obtiene durante las jornadas de trabajo, a tal punto que llega a costarte la vida. Esto también se suele observar en entregas literarias como El corazón de las tinieblas del autor Joseph Conrad (1899) y no suele ser tan ajeno al actual proceso de explotación inmerso en la violencia, el choque de culturas y la discriminación racial, los cuales, a su vez, van de la mano de una concepción desproporcionada de la autorrealización con alto enfoque en la estética. Siendo ejemplo de ello, la industria publicitaria, cuyos paradigmas imperantes suelen ser un reflejo de lo que se considera estético en esos tiempos (Eco, 2005). Nosotros nos hallamos en constante inmersión y explotación, hecho que es graficado en la cinta Sobreviven del director John Carpenter (1988).

No estamos tan ajenos a una realidad similar, tal como lo plantea Peirano (2019) al retratar un escenario en el que el uso de herramientas conductuales permite la perpetuación del ciclo de consumo del público objetivo. Un pilar relevante para el desarrollo de una economía basada en la atención y donde los niveles de pertenencia y afiliación hacia ciertos estímulos obtienen una gran connotación en la esfera social del ciberespacio. Un entorno donde estamos cada vez más conectados a diversos contextos, pero distantes en cuanto a nuestros estilos de vida y consumo responsable. Todo ello, en aras de obtener recompensas inmediatas que fungen como un soma artificial que nos genera un bienestar constante y alimenta el hedonismo propio del ego humano.

\section{Palabras finales}

En síntesis, si bien el Transhumanismo trae consigo diversos paradigmas relevantes en cuanto al desarrollo social, científico y tecnológico, resulta necesario apreciar la otra cara de la moneda, la de una posible realidad alternativa que por más variable, ficticia y lejana que nos parezca, no resulta ser del todo ajena a nosotros. Ya que el arte, así como sus diversas manifestaciones, entre ellas la literatura y cinematografía, demuestran la perspectiva del contexto en la cual fueron elaborados y los posibles escenarios hacia donde podríamos ir o que incluso podríamos estar viviendo en carne propia. Puesto que los límites entre la realidad tecnológica y la ciencia ficción, suelen ser tenues.

\section{Referencias}

Asimov, I. (2002). La Fundación. Plaza \& Janes.

Atwood, M. (2019). El cuento de la criada. Salamandra.

Bacigalupi, P. (2011). La chica mecánica. Plaza y Janés.

Blomkamp, N. (Director). (2015). Chappie [Película]. Media Rights Capital.

Bradbury, R. (2019). Fahrenheit 451. DeBolsillo.

Cárdenas, R. (2019). CRISPR y el nacimiento en china de dos niñas editadas genéticamente Lima: Instituto de

Extrapolítica y Transhumanismo.

https://extrapolitica.ssh.org.pe/wp-

content/uploads/2019/09/C\%C3\%A1rdenas-Krenz-RonaldCRISPR-y-ni\%C3\%B1as-editadas-gen\%C3\%A9ticamenteIET.pdf

Carpenter, J. (Director). (1988). Sobreviven [Película]. Alive Films. 
Conrad, J. (2010). El corazón de las tinieblas. El Comercio.

Dick, P. (2006). ¿Sueñan los androides con ovejas eléctricas? Edhasa.

Eco, U. (2005). Historia de la belleza. Lumen.

García, E. (2009). The repossession mambo. Harper Collins Publishers.

GBD 2019 Universal Health Coverage Collaborators (2020). Measuring universal health coverage based on an index of effective coverage of health services in 204 countries and territories, 1990-2019: a systematic analysis for the Global

Burden of Disease Study 2019. Lancet, 396 (10258), 12501284.https://doi.org/10.1016/S0140-6736(20) $30750-9$

Gibson, W. (2002). Neuromante. Minotauro

González, M. \& García, J. (2006). Arquitecturas emocionales en Inteligencia Artificial: una propuesta unificadora. Teoría de la Educación: educación y cultura en la sociedad de la información, 7 (2), 156-168. https://gredos.usal.es/handle/10366/56510 Huxley,A. (2018). Un mundo feliz. DeBolsillo.

Intergovernmental Panel on Climate Change. (2021). Climate Change 2021: The Physical Science Basis. Contribution of Working Group I to the Sixth Assessment Report of the Intergovernmental Panel on Climate Change, Summary for Policymakers. https://www. ipcc.ch/report/ar6/wg1/

Mendoza, D. (2020). Racismo y roles de género, conductas perpetuadas en algoritmos de inteligencia artificial. Coloquio, 65 (2020),132-135.

Niccol, A. (Director). (2011). El precio del mañana [Película]. Regency Enterprises.

Niccol, A. (Director). (1997). Gattaca [Película]. Columbia Pictures. Orwell, G. (2020). 1984. DeBolsillo.

Orwell, G. (2000). Rebelión en la granja. El Comercio.

Páez, A. \& Ramírez, N. (2020). Análisis jurídico de la discriminación algorítmica en los procesos de selección laboral. Ediciones Uniandes. https://philpapers.org/rec/PEZAJD
Parsons T. (2017). Cyberpsychology and the brain. Cambrigde University Press.

Peirano, M. (2019). El enemigo conoce el sistema. Debate.

Pfister, W. (Director). (2014). Transcendence: Identidad virtual [Película]. Alcon Entertainment.

Radford, M. (Director). (1984). 1984 [Película]. Virgin.

Sánchez, H. \& Sánchez, M. (2017). Los bots como servicio de noticias y de conectividad emocional con las audiencias. El caso de Politibot. Doxa comunicación, 25 (2017), 63-84. https://repositorioinstitucional.ceu.es/handle/10637/8765

Sapochnik, M. (Director). (2010). Los recolectores [Película]. Universal Pictures.

Schlöndorff, V. (Director). (1990). El cuento de la criada [Película]. Bioskop Film.

Scott, R. (Director). (1982). Blade Runner [Película]. The Ladd Company.

Singh, T. (Director). (2015). Inmortal [Película]. Focus Features.

Stephenson, J. (Director). (1999). Rebelión en la granja. [Película] Hallmark Entertainment.

Turchin, A. \& Denkenberger, D. (2018). Classifcation of global catastrophic risks connected with artifcial intelligence. Al \& Society, 35(1), 147-163. https://link.springer.com/article/10.1007/s00146-018-0845-5

Truffaut, F. (Director). (1966). Fahrenheit 451 [Película]. Anglo Enterprises.

Verne, J. (2011). De la tierra a la luna. San Marcos.

Verne, J. (2009). 20000 leguas de viaje submarino. Chirre S.A.

Wolinsky, H. (2016). The FBI and biohackers: an unusual relationship. EMBO reports, 17(6), 793-796. https://www.ncbi.nlm.nih.gov/pmc/articles/PMC5278613/

\section{Cómo citar este artículo:}

Siancas, W. (2021). Transhumanismo Lado B: ¿La religión del futuro? Futuro Hoy, $2(3), 36-39$. https://doi.org/10.52749/fh.v2i3.6

Esta obra está bajo licencia internacional

Creative Commons 4.0 Reconocimiento 4.0. 